\section{Discussion}

Mental health service provision in Malawi is critically low, with 4.5 full-time psychiatrists in the country and 2.5 psychiatric nurses per 100000 people (compared with 11 psychiatrists and 104 nurses per 100000 people in the UK) (Jacob et al, 2007). SMMHEP aims to address the treatment gap through education of students, staff and schools, and has helped produce three new Malawian psychiatry trainees.

Delivery of the new educational course was a positive experience for the volunteers but did present some challenges. Resources, such as textbooks, were limited and there were frequent power cuts. Practical difficulties during the Zomba residential attachment included the student accommodation, which lacked mosquito nets and cooking facilities.

Although this evaluation is of a relatively small number of students, there was a $100 \%$ return rate of questionnaires, showing that the new undergraduate programme, which includes a residential psychiatric hospital attachment, is a valuable and effective way for students to gain knowledge and skills. It also has a direct effect on reducing stigma and improving attitudes to psychiatric illness and psychiatry as a potential career path. The results of this survey appear positive and similar studies in Malawi support the finding that education improves attitudes to psychiatry (Beaglehole et al, 2008).

However, the reality is that although medical students would consider pursuing psychiatry, this interest is not translated into actual careers. In fact, only five graduates have chosen this path since the
College first opened and postgraduate training continues to struggle to recruit people to specialise in psychiatry. It is therefore clear that ongoing work, development and support are required from SMMHEP and the College of Medicine to engage and encourage students to work in psychiatry in the future.

\section{References}

Baig, B. J., Beaglehole, A., Stewart, R. C., et al (2008) Assessment of an undergraduate psychiatry course in an African setting. BMC Medical Education, 8, 23.

Beaglehole, A. L., Baig, B. J., Stewart, R. C., et al (2008) Training in transcultural psychiatry and delivery of education in a low-income country. Psychiatric Bulletin, 32, 111-112.

Bowie, C. (2006) The burden of disease in Malawi. Malawi Medical Journal, 18, 103-110.

Crabb, J., Stewart, R. C., Kokota, D., et al (2012) Attitudes towards mental illness in Malawi: a cross-sectional survey. BMC Public Health, 12,541

Hanlon, C., Fekadu, D., Sullivan, D., et al (2006) Teaching psychiatry in Ethiopia. International Psychiatry, 3, 43-45.

Jacob, K. S., Sharan, P., Mirza, I., et al (2007) Mental health systems in countries: where are we now? Lancet, 370, 1061-1077.

Kauye, F. (2008) Management of mental health services in Malawi. International Psychiatry, 5, 29-30.

Kulhara, P. \& Avasthi, A. (2007) Teaching and training in psychiatry in India: potential benefit of links with the Royal College of Psychiatrists. International Psychiatry, 4, 31-32.

Mullick, M. (2007) Teaching and training in psychiatry and the need for a new generation of psychiatrists in Bangladesh: role of the Royal College of Psychiatrists. International Psychiatry, 4, 29-30.

Saraceno, B., van Ommeren, M., Batniji, R., et al (2007) Barriers to improvement of mental health services in low-income and middleincome countries. Lancet, 370, 1164-1174.

World Health Organization (2005) Mental Health Atlas. WHO
RESEARCH PAPER

\title{
Hospital doctors' management of psychological problems at a Nigerian tertiary health institution
}

\author{
Baba A. Issa, ${ }^{1}$ Abdullahi D. Yussuf, ${ }^{2}$ Olatunji A. Abiodun ${ }^{3}$ and \\ Ganiyu T. Olanrewaju ${ }^{4}$
}

${ }^{1}$ Consultant Psychiatrist, University of Ilorin Teaching Hospital, Ilorin, Nigeria, email issababa2002@yahoo.com

${ }^{2}$ Consultant Psychiatrist, University of Ilorin Teaching Hospital, Ilorin, Nigeria

${ }^{3}$ Consultant Psychiatrist, University of Ilorin Teaching Hospital, Ilorin, Nigeria

${ }^{4}$ Consultant Psychiatrist University of Ilorin Teaching Hospital, Ilorin, Nigeria
A questionnaire was sent to all consenting doctors at the University of Ilorin Teaching Hospital, Nigeria. It asked about their management of psychological problems in their clinical practice. Over $90 \%$ would welcome more time to talk to patients and agreed that psychological and social factors should be routinely assessed and recorded for patients. Most respondents would refer patients with depression or disturbed behaviours. 'Ineffective treatment' and 'dislike of psychiatric referral' were not the main reasons for non-referral. A majority of the doctors had initiated treatment for anxiety and insomnia but not for alcohol withdrawal, psychosis, acute confusional state or depression. Doctors' awareness of 'the impact of psychological factors on the course of physical illness' was high. To sustain this high level of awareness and encourage referral, in-house psychoeducational training of hospital doctors should be intensified. In addition, an increased doctor/patient ratio, public education to reduce stigma and a well developed liaison psychiatric service are imperative. 
We are grateful to all the staff of UITH, Ilorin, who participated in the study, for their patience and cooperation during the period of data collection.
Adequate management of psychological problems by hospital doctors (other than psychiatrists) can determine the effectiveness (or otherwise) of liaison psychiatric services in the hospital (Deventer et al, 2008). Hospital doctors should aim to ameliorate seemingly minor but incapacitating psychiatric disorders and symptoms. Many patients who require psychiatric services are deprived of them because of non-recognition of their psychological problems by attending physicians and lack of referral (Dixon et al, 2001; Bartels et al, 2005). This can prolong suffering and lead to a deterioration of the patient's clinical status and to complications. Globally, psychological problems account for a significant proportion of disease morbidity and mortality, and have taken a significant toll on time lost due to morbidity, in terms of disability-adjusted life years (DALYs) (World Health Organization, 2012). Therefore, it is of interest to determine how psychological problems are managed. According to Faizen et al (2012), the attitude to psychiatry of non-psychiatrists and non-psychiatry resident doctors is crucial because of the large number of psychiatric patients who will present to them, directly or indirectly.

Morgan \& Killoughery (2003) revisited a study by Mayou \& Smith (1986) on hospital doctors' management of psychological problems in London hospitals. In their study, more hospital doctors were aware of the psychological needs of patients (Morgan \& Killoughery, 2003). There appeared to have been improvement in liaison psychiatric services, as evidenced by an increase in the number of qualified psychiatrists at public health institutions in Nigeria. This improvement was expected to translate to more shared knowledge between mental and other health practitioners and better mental health services. Some doctors, however, although familiar with mental health issues, are likely not be too different from non-doctors in stereotyping and stigmatising mental illness. The negative attributes of these doctors could affect their practice when they encounter individuals with mental illness (Faizen et al, 2012).

The present study set out to replicate these previous studies, using the same methods in Nigeria, in order to ascertain whether a supposed increase in awareness of mental health issues and the introduction of liaison psychiatry had had any impact on the management of mental health conditions by hospital doctors.

\section{Method}

The data for this study were collected as part of a larger study to determine hospital doctors' management of mental illness and the psychological impact of their work on the doctors, particularly in terms of their alcohol use (Issa et al, 2012). The study was carried out at the University of Ilorin Teaching Hospital (UITH), a tertiary centre that provides health services for Kwara State and surrounding states (Nigeria is a federation of 36 states). Questionnaires together with information sheets and consent forms were distributed to all doctors in the service of the hospital except those in the department of behavioural sciences. One questionnaire covered: sociodemographic information (age, gender, marital status); professional qualification; receipt of treatment for any form of emotional disturbance by the participants or their relatives; and working conditions (e.g. membership of workplace leisure or social clubs, perceived cordiality with co-workers and patients, perception of workload, and satisfaction with remuneration). They also completed a questionnaire (the same used by Mayou \& Killoughery) on their management of psychological problems in their clinical practice, on which this report is based.

The ethics and research committee of the hospital approved the study protocol.

\section{Results}

Questionnaires were distributed to 350 doctors: 100 house officers, 150 resident doctors and 100 senior doctors (i.e. medical officers and consultants). Responses were received from 241 (68.9\%), of whom $134(55.6 \%)$ were aged 35-45 years, 182 $(75.5 \%)$ were male, and $202(83.8 \%)$ were married. The largest number of respondents were from the department of internal medicine (35 or $14.5 \%$ ), followed by department of surgery (32 or 13.3\%).

About half (109 or $45.2 \%$ ) of the respondents had 3-10 years' working experience. About threequarters (190 or $78.8 \%$ ) had had 4-8 weeks of exposure to mental health training at undergraduate level, while $181(75.1 \%)$ had had no exposure at postgraduate level. Most of the respondents (221 or $91.7 \%$ ) had no personal history of mental illness and the majority had no family history of it (180 or $74.7 \%$ ).

\section{General attitudes to mental illness and medical responsibility for its management}

Over half of the respondents believed that 'emotional and social aspects of care enhanced their job interest' while less than $40 \%$ agreed that 'management of emotional issues is solely a medical responsibility' (Table 1).

About a quarter of the doctors agreed that they have major roles in the management of depression (Table 2). Just under half would take responsibility for the management of acute confusional state, while over two-thirds would take responsibility for drug overdose, a fifth for chronic drinking problems, less than a fifth for disturbed behaviours but over half for the emotional care of dying patients.

\section{Time constraints and assessments of psychological problems}

Over $90 \%$ of the doctors would 'welcome more time to talk to the patients' and agreed that 'psychological and social factors should be routinely assessed and recorded for patients' (Table 1). However, about a quarter of the doctors agreed that it was 'impractical for hospital doctors to assess and treat emotional problems', and on this item there was the largest difference between the 
Table 1

Questionnaire responses in the present study and two comparison studies: percentage agreement with statements

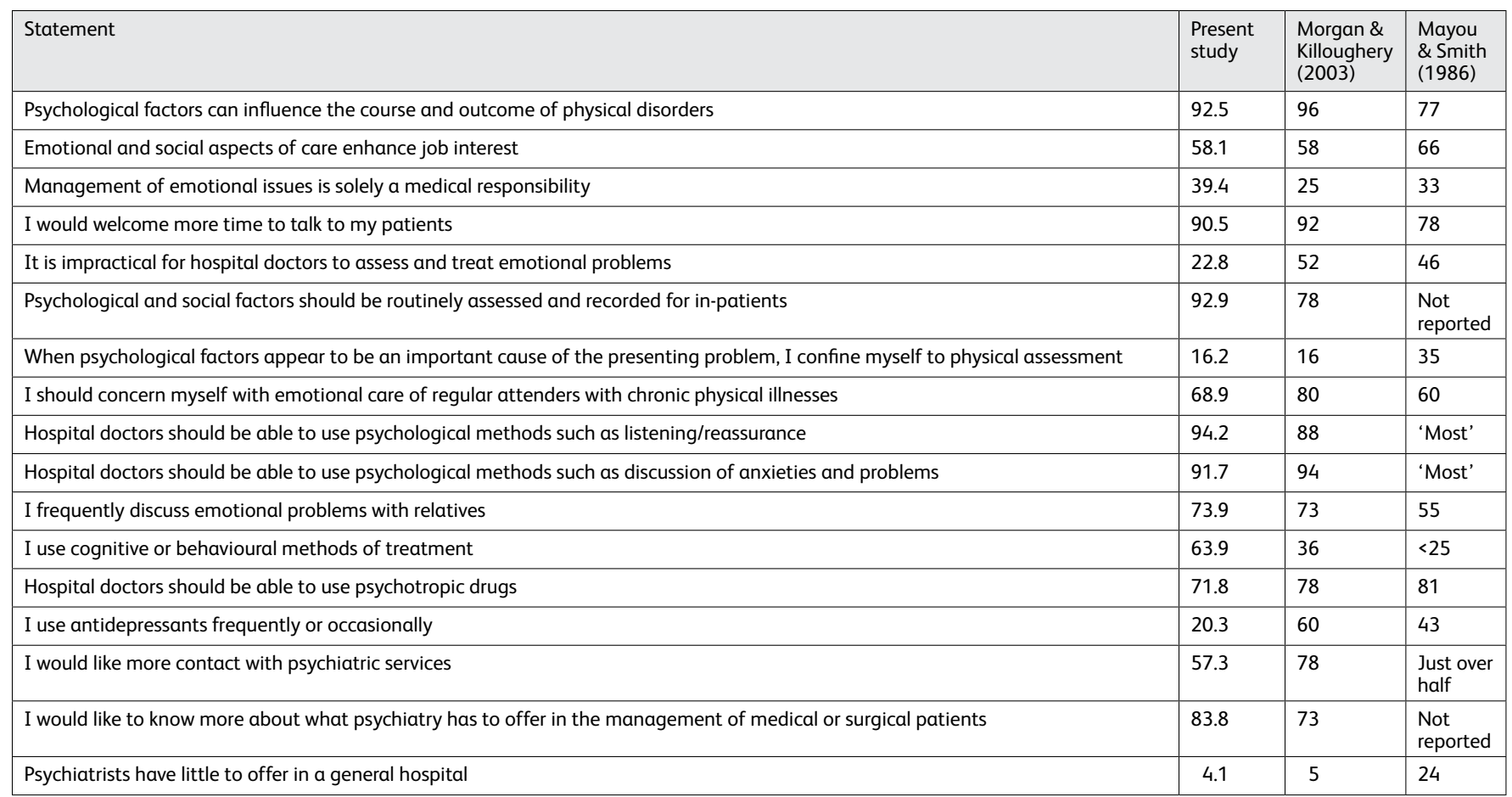

\section{Table 2}

Agreement with statements that major responsibility for common types of psychological problems lies with hospital doctors (other than psychiatrists): percentage of the present sample $(n=228)$, with responses from Morgan \& Killoughery (2003) and Mayou \& Smith (1986) respectively in parentheses

\begin{tabular}{|l|l|l|l|l|}
\hline & Strongly agree & Agree & Uncertain & Disagree \\
\hline Depression & $4.4(10,5)$ & $20.6(21,21)$ & $4.4(10,20)$ & $49.1(49,41)$ \\
\hline Acute confusional state & $4.4(27,17)$ & $42.5(51,59)$ & $11.8(7,13)$ & $32.5(9,10)$ \\
\hline Overdoses & $16.2(24,7)$ & $55.7(37,29)$ & $9.2(9,33)$ & $15.8(19,27)$ \\
\hline Chronic drinking problems & $5.7(6,5)$ & $14.9(24,20)$ & $8.8(18,33)$ & $43.0(36,38)$ \\
\hline Disturbed behaviour & $7.0(4,3)$ & $9.6(19,25)$ & $6.6(23,35)$ & $42.5(34,34)$ \\
\hline Emotional care of dying patients & $15.4(15,10)$ & $43.2(59,23)$ & $12.8(14,26)$ & $25.6(8,37)$ \\
\hline
\end{tabular}

present findings and those of the two earlier (UK) studies. In the present survey, $16.2 \%$ of doctors would 'confine themselves to physical assessment only' even when psychological factors appeared to be an important cause of the presenting problem.

\section{Attitudes to psychiatry, psychiatric treatments and barriers to referral}

A large majority of the respondents agreed that hospital doctors should be able to use psychological methods such as 'listening/reassurance' and discussion of anxieties and problems (Table 1). About three-quarters agreed that 'hospital doctors should be able to use psychotropic drugs', but only $20 \%$ claimed to 'use antidepressants frequently or occasionally'. Very few respondents agreed with the statement 'Psychiatrists have little to offer in a general hospital', while more than half would like 'to have more contact with psychiatric services'. In addition, over $80 \%$ would 'like to know more about what psychiatry has to offer in the management of medical and surgical patients'.
Almost all the respondents would refer patients with depression (96.5\%), disturbed behaviours $(96.5 \%)$; dementia $(67.8 \%)$ or acute confusional state $(69.6 \%)$. However, significant numbers would 'not refer a patient because of a diagnostic problem' $(60.5 \%)$ or 'non-compliance with treatment' $(60.2 \%)$.

Only $9.7 \%$ of respondents gave 'ineffective psychiatric treatment' as the reason for non-referral of patients, and only $25.3 \%$ gave 'dislike for psychiatric referral', but $48.8 \%$ said it would be because of 'stigmatisation'. A substantial number of the doctors had initiated treatment in anxiety disorders (57.4\%) and insomnia (73.3\%), but most had not initiated treatment in alcohol withdrawal $(91.5 \%)$, psychosis $(88.1 \%)$, acute confusional state $(78.6 \%)$ and depressive disorders (75.0\%).

\section{Discussion}

A response rate of $68.8 \%$ was considered reasonable, considering the anonymous nature of the study, which seemed to have reduced 
researcher-influenced bias in completing the questionnaire.

The relatively few years of practice of most of the respondents in this study is likely explained by the younger ages of our respondents (most were under 45 years old). Traditionally in Nigeria, candidates are admitted to medical school after their secondary school education, unlike in some countries where a university degree is a prerequisite for admission. Thus, these candidates graduated as doctors while still young.

The Nigeria medical curriculum is designed to expose medical students to a minimum of 4 weeks of mental health education and clerkship. Graduate doctors therefore might not have acquired adequate training in basic mental health, which could adversely affect their practice. The postgraduate residency training that could further expose these doctors to mental illness is provided for only a few postgraduate specialties. For example, surgery and surgical subspecialties do not rotate through the psychiatric department during their residency training, although medically related specialties such as internal medicine and family medicine do include such postings. This gap in training has the potential to adversely affect mental health practice and management among these doctors after their qualification.

In this study a majority of the respondents did not report a history of psychiatric illness among themselves or their relatives. Similarly low frequencies of reported mental illness have been documented in previous studies (Phillips et al, 2000; Saunders, 2003) and this has been largely attributed to stigma (Saunders, 2003; Westbrook, 2011).

\section{The management of common psychological problems}

Awareness of the impact of psychological factors on the course of physical illness was high and comparable to the British studies. The doctors in this study, similarly to the previous studies (Mayou \& Smith, 1996; Morgan \& Killoughery, 2003), recognised that psychological factors could influence the course and outcome of physical disorders. This could be described as being good for mental health and its practice because the psychological health of physically ill patients is then adequately considered in the management of such physical illnesses, more especially if more time can be devoted to these patients. Similarly, well over half the doctors agreed that emotional and social aspects of care enhanced their job interest, as in the previous studies. Most of the doctors did not agree that the management of emotional issues was solely a medical responsibility.

The positive responses observed in this study could have resulted from medical training (both undergraduate and postgraduate) or from the positive impact of liaison psychiatric services.

A majority of the respondents in this study would 'rather not leave the care of depression to psychiatrists'. Over two-thirds disagreed that hospital doctors (other than psychiatrists) have the major responsibility for the management of depression (higher than in the British studies). In contrast, a majority agreed that non-psychiatrists have the major responsibility for overdose and for the emotional care of dying patients, but not for disturbed behaviours or chronic drinking problems, which were predominantly regarded as being in the realm of psychiatrists.

The reason for around half of the hospital doctors agreeing that they have the major responsibility for the management of acute confusional state could be that most causes of this disorder are physical, such as infection, surgical emergencies, head injuries and medication side-effects. This may reflect the traditional mind/body dichotomy, whereby medical disorders are regarded as diseases of the mind or body (Mehta, 2011).

\section{Time constraints}

As in the previous studies (Mayou \& Smith, 1986; Morgan \& Killoughery, 2003), a majority of the doctors would welcome more time to talk to their patients. This supports the notion that time constraints are a reason for non-psychiatrist doctors not making adequate diagnosis of psychological problems. Time constraints have been identified as a major factor preventing the diagnosis of mental disorder by general practice doctors (Alexander \& Fraser, 2008).

A solution to this problem might be proper time management, because most of the doctors agreed that they should provide emotional care for regular attenders with chronic physical illnesses. Similarly, most did not agree that when psychological factors appeared to be an important cause of the presenting problem they would nonetheless confine themselves to physical assessment. Such assertions seemed to indicate the readiness of these doctors to participate in the assessment and treatment of patients with psychological problems and physical disorders. A psychoeducational programme aimed at improving the diagnosis and management of psychological problems by non-psychiatrist doctors and the use of short diagnostic instruments (to reduce evaluation time) would go a long way to overcoming time constraints.

A contributory factor is the inadequate doctor/ patient ratio in low-income countries like Nigeria. In the year 2005, the doctor/patient ratios in Nigeria, Ghana, Kenya and South Africa were 28, 15,14 and 77 per 100000 population, and 151 and 134 in Seychelles and Tunisia, but about 333 per 100000 population in Germany and France (United Nations Development Programme, 2006). A lower ratio would help overcome the time constraints reported by this cohort of Nigerian hospital doctors.

\section{Attitude to psychiatry and barriers to referrals}

Similar to the previous studies, all aspects of talking therapies were endorsed by the doctors, thus indicating positive (or improved) attitudes of the hospital doctors to patients who are 
mentally ill. Caution is needed here, however, as hospital doctors still require training on the use of psychotherapeutic methods. While listening and reassurance are skills that all doctors must possess, the use of cognitive and behavioural methods (claimed to be used by nearly two-thirds of respondents) should be reserved for specialists (i.e. psychotherapists). In fact, the use of such methods in previous studies (Mayou \& Smith, 1986; Morgan \& Killoughery, 2003) was much less impressive than the level reported in this study. A reason for this could be that the respondents in this study did not comprehend what was meant by cognitive and behavioural methods, as formal cognitive and behavioural therapies are not provided at the study centre. Contrary to this was the use of psychotropic drugs. As in the previous studies (Mayou \& Smith, 1986; Morgan \& Killoughery, 2003), respondents agreed that hospital doctors should be able to prescribe psychotropic drugs. While one would agree with the doctors on this unrestricted prescription of psychotropic drugs, this has to be done with extreme caution, so as to prevent dependency and tolerance (and any resultant iatrogenic psychological problems). The low proportion of respondents prescribing antidepressants (frequently or occasionally) was not surprising, given that, when compared with the two previous studies (Mayou \& Smith, 1986; Morgan \& Killoughery, 2003), a much smaller proportion agreed that hospital doctors had the major responsibility for the treatment of depression.

The cohorts' attitude to psychiatry was, however, encouraging. Their readiness to have more contact with psychiatric services and eagerness to know more about what psychiatry has to offer in the management of medical and surgical patients were satisfying. These attributes need to be reinforced for a more holistic management of patients with physical disorders. Psychiatrists have more responsibilities in general hospital settings in this regard.

Despite the readiness of the doctors to refer patients, many were not referring because of the perceived stigma.

\section{Limitations of the study}

Being a self-reported questionnaire study, it is not possible to determine whether the questionnaire responses accurately reflected actual clinical practice, and the study design was subject to social desirability bias (Morgan \& Killoughery, 2003). However, the same questionnaire was used in the two British comparison studies.

\section{Conclusion}

The doctors in this study, similar to those in the previous studies, recognised that psychological factors could influence the course and outcome of physical disorders. Many were ready to take responsibility for the care of some organic mental disorders, such as acute confusional state, but much less so depression and disturbed behaviours. The respondents were, though, more receptive to 'assessing and treating emotional problems' than in the two British studies. Time constraint was recognised as a major impediment to the diagnosis of psychological disorders but the doctors expressed readiness for more contact with psychiatric services. While willing to refer patients with psychological problems to psychiatrists, stigma was identified as a barrier. Therefore, we suggest that in-house psychoeducational training of hospital doctors should be intensified. The training could include the use of brief diagnostic tools that would shorten the time taken to make diagnoses. Such training might also help disseminate information to the doctors on the use of psychotropic drugs and psychological therapies. Improving the low doctor/patient ratio is also advised. Addressing these issues could help ameliorate the problem of time constraint.

Lastly, well developed liaison psychiatric services where psychiatrists are incorporated within medical or surgical teams could help to solve the problems hampering referral for psychiatric evaluation.

\section{References}

Alexander, C. \& Fraser, J. (2008) General practitioners' management of patients with mental health conditions: the views of general practitioners working in rural north-western New South Wales. Australian Journal of Rural Health, 16, 363-369

Bartels, S. J., Blow, F. C., Brockmann, L. M., et al (2005) Substance Abuse and Mental Health Care Among Older Americans: The State of Knowledge and Future Directions. WESTA.

Deventer, C. V., Couper, I., Wright, A., et al (2008) Evaluation of primary mental health care in North West province a qualitative view. South African Journal of Psychiatry, 14, 136-140.

Dixon, L., Green-Paden, L., Delahanty, J., et al (2001) Variables associated with disparities in treatment of patients with schizophrenia and comorbid mood and anxiety disorder. Psychiatric Services, 52, 1216-1222.

Faizen, S., Raveesh, B. N., Sujatha, R. L., et al (2012) Attitude of non-psychiatry doctors to psychiatry and its correlates to in Mysore South India. BMC Proceedings, 6 (suppl. 4), 14

Issa, B. A., Yussuf, A. D., Abiodun, O. A., et al (2012) Hazardous alcohol use among doctors in a Nigerian tertiary hospital. West African Journal of Medicine, 3, 97-101.

Mayou, R. \& Smith, E. B. O. (1986) Hospital doctors' management of psychological problems. British Journal of Psychiatry, 148 194-197.

Mehta, N. (2011) Mind-body dualism: a critique from a health perspective. Mens Sana Monographs, 9, 202-209.

Morgan, F. \& Killoughery, M. (2003) Hospital doctors' management of psychological problems - Mayou \& Smith revisited. British Journal of Psychiatry, 182, 153-157.

Phillips, M. P., Pearson, V., Li, F., et al (2000) Stigma and expressed emotion: a study of people with schizophrenia and their family members in China. British Journal of Psychiatry, 181, 488-493.

Saunders, J. C. (2003) Families living with severe mental illness: literature review. Issues in Mental Health Nursing, 24, 175-198.

United Nations Development Programme (2006) Doctor to patient ratio in Africa. Available at http://hdr.undp.org/hdr2006/statistics/ indicators/58.html (accessed 17 May 2013).

Westbrook, A. H. (2011) Mental health legislation and involuntary commitment in Nigeria: a call for reform. Washington University Global Studies and Law Review, 39. Available at http:// http:// digitalcommons.law.wustl.edu/globalstudies/vol10/iss2/7 (accessed 23 August 2011).

World Health Organization (2012) Global health risks: mortality and burden of disease. Available at http://www.who.int/healthinfo/ global_burden_disease/GlobalHealthRisks_report_full.pdf (accessed 11 October 2012) 